\title{
Targeted Molecular Imaging
}

\section{E. Edmund Kim, MD}

\section{Index terms:}

Mulecular analysis

Radiolgy and radiologists, research

Review

Korean J Radiol 2003; 4:201-210

Received August 19, 2003; accepted after revision November 9, 2003.

Department of Radiology and Medicine, Center for Metabolic Imaging, Nuclear Medicine, The University of Texas MD Anderson Cancer Center, Houston, TX 77030 USA.

Address reprint requests to:

E. Edmund Kim, MD, Center for Metabolic Imaging, Nuclear Medicine, Box 59 Houston, TX 77030 USA.

Telephone: (713) 794-1052

Fax: (713) 794-5456

e-mail: ekim@di.mdacc.tmc.edu
Molecular imaging aims to visualize the cellular and molecular processes occurring in living tissues, and for the imaging of specific molecules in vivo, the development of reporter probes and dedicated imaging equipment is most important. Reporter genes can be used to monitor the delivery and magnitude of therapeutic gene transfer, and the time variation involved. Imaging technologies such as micro-PET, SPECT, MRI and CT, as well as optical imaging systems, are able to non-invasively detect, measure, and report the simultaneous expression of multiple meaningful genes. It is believed that recent advances in reporter probes, imaging technologies and gene transfer strategies will enhance the effectiveness of gene therapy trials.

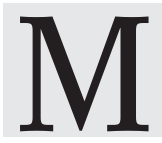

olecular imaging is a technique that uses molecules or generates signals from molecules to image the structure and function of molecular systems (1). The procedure may involve proteins, cellular subassemblies, cells, organ systems, or the whole organism, but in each case the image obtained displays specific molecular components of structures or molecular functions.

Molecular medicine represents the merger of modern biology and medicine. Diseases result from internally and externally derived molecular errors that instruct cells to transform into the phenotypes of disease; the genome coded by four nucleotides to yield instructions is translated into another code assembly by combinations of 20 amino acids to form proteins upon which the structure and function of cells are then based.

Advances in molecular medicine are associated with one wave of technology after another, and range from various biotechnologies to more recent nanotechnologies which are examining how biological systems assemble molecular motors and levers, as well as communicate, compute, and store information. Positron emission tomography (PET) and microPET use positron-labeled molecules to image processes such as metabolism, cell communication, and gene expression. Single photon emission computed tomography (SPECT) and microSPECT systems are used with PET to attain common goals, but employ molecules labeled with single-photon emitters. Optical imaging systems use fluorescence or bioluminescence to image gene expression in cells through the use of reporter genes that yield a protein which emits.

Structural imaging systems have been fused with molecular imaging technologies, and such mergers include PET/ computed tomography (CT), SPECT/CT, optical fluorescence imaging (used to obtain anatomical photographs), magnetic resonance imaging (MRI), functional MRI, and magnetic resonance spectroscopy using molecular imaging probes. Nuclear imaging benefits greatly from molecular probes originating from biochemistry and pharmaceuticals with relatively poor spatial resolution. Other 
imaging approaches suffer from nonspecificity (CT), low signal penetration through tissue (optical), or the low signal strength from specific molecular constituents (MRI).

This paper briefly reviews the nature of genes, gene therapy and imaging, as well as the imaging of angiogenesis and apoptosis.

\section{DNA, Genes and Gene Therapy}

The genetic information needed to sustain life is contained in the DNA molecule, which is composed of four chemical bases: adenine, thymine, cytosine and guanine. Strands of these bases pair with each other to form a chromosome around histone proteins. The nucleus of a human cell contains 23 chromosome pairs, and is referred to as the human genome, which contains approximately 60,000100,000 genes; a single gene is a stretch of DNA and consists of several thousand base pairs. A new molecule, RNA, is synthesized by using DNA as a template when a gene becomes active; the RNA acts as a template for the protein. Approximately 80,000 proteins perform all life-related functions the inside and outside cells; to identify a function, a knowledge of the sequence of the human genome is required. In order to identify new targets for the earlier detection of diseases, to evaluate molecular markers for therapeutic responses, and use imaging for drug selection and gene expression, the human genome project will determine the exact sequence of 3 billion base pairs in human DNA.

If a particular gene has mutated, it may not be able to produce protein or it may function poorly or too aggressively. This disrupts cell and tissue functions and causes abnormal cell behavior, leading to symptoms of diseases. Gene therapy involves the deliberate transfer of DNA for the correction of mutated genes through the introduction of a normal gene into the cell or by direct repair (3). Viruses are ideal vectors, being naturally suited to the delivery of genetic material into target cells. RNA viruses, such as retroviruses, reverse the normal sequence of events by converting their RNA genome back into DNA by using reverse transcriptase, and the DNA thus produced is integrated into the genome. A DNA coding sequence is derived from cellular messenger RNA, paving the way for gene cloning (4). Reverse transcriptase synthesizes a second complementary DNA (cDNA) strand, a process which yields a double-stranded DNA molecule.

Polymerase chain reaction is the method used fo produce amplifying cloned DNA in sufficient quantities. After cloning in the expression vector, transfection into bacteria allows the large-scale manufacture of milligram-to-gram quantities of the cDNA-vector complex. The cloned cDNA must be purified from the bacteria, after which it is suitable for transfer into the gene delivery vehicle. Genes in the form of DNA are delivered to a target cell by packing them in a virus, applying them in the form of plasmid or plasmid in a synthetic delivery system, or delivering them through direct injection, electroporation, gene guns, or radiofrequency pulses. Once inside the cells, transgenes can be found within the cytoplasm or in the nucleus, or may be integrated into the host genome. The duration and level of foreign gene expression is usually long after the gene is incorporated into the nucleus (5).

Current gene technology has focused on the use of viral vectors which can provide highly efficient transduction and high levels of gene expression (6). Adenoviruses contain double-stranded DNA and enter the cell by receptor-mediated endocytosis. They are quite immunogenic and do not integrate into the host genome (5). Retroviruses contain a single-stranded RNA genome, and enter the cell by direct fusion with the plasma membrane prior to integration into the host chromosome during cell division. They infect only dividing cells however, and become inactivated by serum complement. Herpes simplex virus-derived amplicons have been used as an alternative method of gene delivery. Amplicons are plasmids that can be packaged into recombinant viral particles and used to transfer anticancer genes with little toxicity; these particles are often unstable, immunogenic, and can have lower titers, and oncogene activation may occur, leading to the development of cancer. Transfection methods using nonviral artificial vectors carrying plasmid DNA have therefore been developed; vectors include cationic liposomes, poly-L-lysine-DNA complexes, DNA-coated microprojectiles, dendrimers, and free-plasmid DNA. Although these nonviral vector systems seem to work to some degree ex vivo, gene transfer is generally lower in vivo (7).

In the setting of genetic deficiencies, the goal of therapy is the restoration of deficient or absent expression of a critical gene product. This has been applied to cystic fibrosis (transmembrane conductance regulator), adenosine deaminase deficiency and Gaucher's disease (beta-glucocerebroside deficiency) (8). Chronic pathologic processes such as peripheral vascular disease may be amenable to gene therapy and many gene therapy protocols to date have concentrated on treatments for cancer (9). In general, cancers involve one mutation to a protooncogene (yielding oncogene) and at least one to a tumor suppressor gene, allowing the cancer to proliferate. Gene therapy pursues a variety of strategies, namely oncogene inactivation, tumor suppressor gene replacement, immunopotentiation, molecular chemotherapy, and drug resistance genes. Emphasis will be on genetically modifying cells of the body's own immune system to fight tumors (10). Oncogene activity can be in- 
hibited with antisense RNA, intracellular antibodies, catalytic RNA molecules or antioncoprotein antibodies. Cellular proliferation involves an intricate balance between signals driving cell-cycle progression, those maintaining quiescence, and those initiating the pathway for cellular destruction (i.e., apoptosis). Antibodies directed against fas ligand expressed on the tumor cell may mimic fas/fas ligand interactions and trigger the apoptotic death of the tumor (11). Tumor suppressor gene, p53, plays in regulating tumor cell apoptosis. Retroviral p53 gene complexes injected into refractory p53-deficient lung carcinoma showed tumor regression (12). Expression of p53 is synergistic with cisplatin, and adjacent tumor cells that have not been transduced are also killed by the bystander effect (13). Other tumor suppressor genes include BRCA1, retinoblastoma, and Wilm's tumor genes (14). Passive immunotherapy involves harvesting tumor-infiltrating lymphocytes and treating them to express increased cytokines. Various cytokines including IL-2, IL-4, IL-12, GM-CSF tumor necrosis factor and interferon, have been inserted into tumors and have increased anti-tumor immunity $(15,16)$. Active immunotherapy genetically modifies tumor cells, to increase the expression of antigen-presenting molecules or local concentrations of cytokines; cells are irradiated prior to being returned to the patient. This approach, cancer vaccination, has been the subject of clinical trials for metastatic melanoma (17).

Tumor-lymphocyte interaction is facilitated by co-stimulatory ligand-receptor (B7-CD28) interaction. B7 is absent in many tumors, and re-establishing its expression has been shown to facilitate immune recognition (18). An alternative approach to increasing tumor immunogenicity is to express foreign HLA antigens in the tumor by direct intratumoral transfer which causes the tumor to be rejected by the host immune system (19). Molecular chemotherapy and drug-resistant genes direct chemotherapeutic drug production by the tumor itself. A suicide gene, herpes simplex virus-1 thymidine kinase (HSV1-tk), that encodes a prodrug-converting enzyme, thymidine kinase, is inserted into tumor cells, and a specific nontoxic prodrug (ganciclovir) is then administered systematically (20). The prodrug is converted into a toxic antimetabolite which causes tumor cell death. The cytosine deaminase gene expressed by tumor cells allows 5-fluorocytosine (5-FC) to be converted into 5fluorouracil (5-FU), an antimetabolite; significant anti-tumor activity was reported when a cytosine deaminasebased gene transfer system was used in colorectal cancer (21).

Irrespective of the specific type of delivery vehicle used, the gene must be delivered efficiently to its intended target. Although vectors have been injected systemically, this has proven less efficacious than local delivery. Embolization techniques may aid in prolonging vector contact with the target cells by delaying washout and thereby further enhancing target cell uptake (22). Other delivery strategies include image-guided delivery to target tissues or the tumor through stereotactically placed needles, and cavitary administration using US, CT or MR guidance. Additional interventional strategies have been employed to improve gene delivery, including electroporation, microinjection, particle bombardment, gene gun therapy, and high-frequency ultrasound.

\section{Approaches to Gene Imaging}

Essential to the sampling of molecular information is the use of highly specific imaging probes, sensitive systems producing high resolution images, and appropriate amplification (1). Considerable research efforts are being devoted to the development of suitable in vivo affinity ligands (molecular probes), efficient organ and intracellular targeting, optimal amplification and imaging systems which provide both high resolution and high sensitivity. Current therapeutic drugs are directed against approximately 500 molecular targets (receptors in $45 \%$ of these, enzymes in $30 \%$, and others in $25 \%$ ). In-vivo imaging methods (Table 1) are being developed for gene delivery by using novel formulations of DNA, and for gene expression by using cell-specific, replication-activated, drug-controlled expression systems. Nuclear and optical imaging techniques are $10^{4}-10^{6}$ times more sensitive than MRI or CT for probe detection, but both lack significant spatial resolution. Highresolution 3-D MRI or CT maps of transgene expression can be generated, and a variety of targeted MR or CT contrast agents have been developed for molecular imaging (23). However, if less abundant targets are to be probed for, MRI or CT will require amplification strategies which provide more sensitive detection. In target overexpression, hyperexpression of a targeted molecule results in the static accumulation of contrast agent at the target site. If the targeted gene product is internalized the probed gene product can participate multiple times to sequester targeted contrast agent within the cells. The endogenous reporters exploit the inherent MRI ability of the gene product to enhance MR signal within cells. Tyrosinase expression increased a melanin production, and melanin has a high metal (iron) binding capacity, resulting in high MR signal intensity. A contrast probe can be activated by a cellular protein which can be imaged by utilizing antibodies or ligands to target MR or CT contrast agents to cells. The expression of wild-type transferrin receptor (TfR) correlates with cellular proliferation, and the increased TfR levels found in cancer 
cells may be due to the increased metabolic need for iron, which is essential for cellular metabolism. The applicability of TfR targeted MRI is underway to aid in earlier detection of cancers. MRI techniques have also shown encouraging results using substrate (EgadMe), which can be enzymatically processed by $\beta$ galactosidase and can generate MRI signals. Gliosarcoma cells with transfected human transferrin receptor were imaged using iron oxide nanoparticles conjugated with human holotransferrin. Direct imaging of the expression of therapeutic genes requires the development of many different radiolabeled probes, and thus the development of a more general approach to the indirect monitoring of therapeutic gene expression is needed. Reporter gene imaging is one such potential approach and has been used to study promoter/regulatory elements, inducible promoters, and endogenous gene expression. Reporter gene imaging involves the indirect visualization of transcriptional and post-transcriptional regulation of target gene expression, and of specific intracellular proteinprotein interactions. Regulatory regions of genes (i.e., promoters/enhancers) can be cloned and used to drive transcription of reporter gene. One can indirectly monitor the gene expression by introducing reporter gene with a promoter into the target. Conventional methods to monitor gene expression take advantage of reporter genes including $\beta$ galactosidase (24). Other methods make use of optical reporter genes, including luciferase or green fluorescent protein (GFP) (25). MRI offering higher spatial resolution (micrometer) is more useful than nuclear technique for imaging transgenic animals and cell trafficking, but less sensitive (millimolar rather than picomolar). Near-infrared fluorescence imaging relies on light of a defined band-width as a source of photons that encounter a fluorescent molecule (optical contrast agent) which emits a signal that can be captured with a high sensitivity charge-coupled-device camera. One of the advantages of near-infrared optical imaging is that quenched fluorescent after interaction with their target can be used. It would be suitable to image enzymes, DNA and/or RNA targets. Multiple probes with spectral characteristics can be used for multi- channel imaging. Bioluminescent imaging exploits the emission of visible photons at a specific wave length arising from energydependent reactions catalyzed by luciferases. Expression of the bioluminescent reporter luciferase has been used to image transformed tumor cells and bacterial organisms (1).

Two approaches are possible to adapt the reporter gene concept for PET or SPECT. The reporter gene can be introduced in such a way that it encodes for an enzyme that is capable of trapping a specific tracer through the action of that enzyme. A second approach uses a reporter gene that encodes for an intracellular and/or extracellular receptor capable of binding a tracer. The reporter gene introduced, driven by a promoter of choice, is referred to as a transgene. For all vectors, the common feature is a cDNA expression cassette containing the reporter gene of interest. The promoter can be constitutive, inducible, and also cellspecific. Wild-type HSV1-tk or a mutant HSV1-tk gene, HSV1-sr39tk, are the reporter genes most commonly used in current molecular imaging studies using radiolabeled probes and PET imaging (26).

The use of cytosine cytosine deaminase as a reporter gene, with 5-FC as a reporter probe, has been investigated (27). The cytosine deaminase enzyme converts antifungal agent 5-FC to highly toxic 5-FU which can block DNA and protein synthesis through the substitution of uracil by 5-FU in RNA and the inhibition of thymidilate synthetase by 5 fluorodeoxyuridine monophosphate, resulting in impaired DNA biosynthesis. Another reporter gene system uses the

Table 1. Imaging of Gene Expression

\begin{tabular}{llll}
\hline \multicolumn{1}{c}{ Marker Protein } & Substrate or Ligand & Method & Mechanism \\
\hline $\begin{array}{l}\text { Cell surface } \\
\text { Somatostatin receptor }\end{array}$ & Peptides & Nuclear & Affinity binding \\
Transferrin receptor & Transferrin & MRI & Transchelation \\
Fusion proteins & Oxotechnetate & Nuclear & Phosphorylation \\
Intracellular & & & Deamination \\
Thymidine kinase & FIAU, ganciclovir & Nuclear & Oxidation \\
Cytosine deaminase & Cytosine & Nuclear & Fluorescence \\
Tyrosinase & Tyrosine, DOPA & Nuclear, MRI & Bioluminescence \\
Green fluorescent protein & None & Optical & Fluorescence \\
Luciferase & Luciferin & Optical & Cleavage of galactose \\
Cathepsin & Fluorochromes & Optical & Trapping and organification \\
Sgalactosidase & Galactosylated chelators & MRI & Nuclear \\
Sodium iodide symporter & lodine &
\end{tabular}

Note. - FIAU = 2'-fluoro-2'-deoxy-1- $\beta$ D-arabinofuranosyl-5-iodo-uracil, DOPA = dioxyphenyl-alanine 
dopamine type 2 receptor $\left(\mathrm{D}_{2} \mathrm{R}\right)$, which binds spiperone, a $\mathrm{D} 2 \mathrm{R}$ antagonist. The presence of F-18 fluorethyl spiperone (FESP) results in probe accumulation in D2R-expressing cells or tissue (28). The expression of HSV1-sr-39 tk and $\mathrm{D}_{2} \mathrm{R}$ receptor genes can be imaged simultaneously with mi- cro PET by using F-18 fluoropenciclovir and F-18 FESP (29).

Somatostatin receptor subtype II (SSTR2) is another reporter gene, and through the use of In-111, I-123 or Tc99m-labeled octreotide, SSTR2-positive neuroendocrine

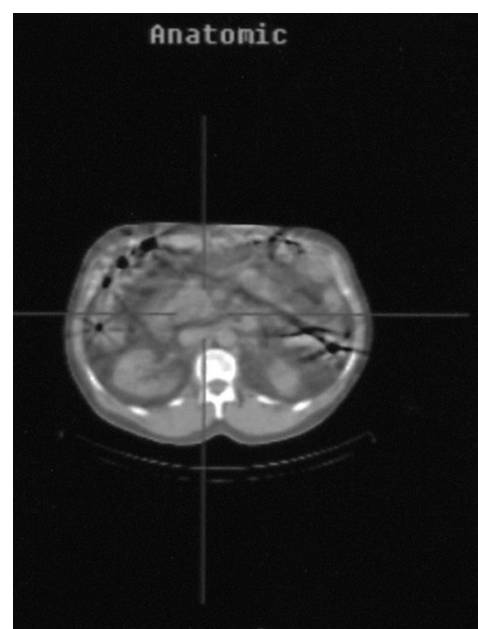

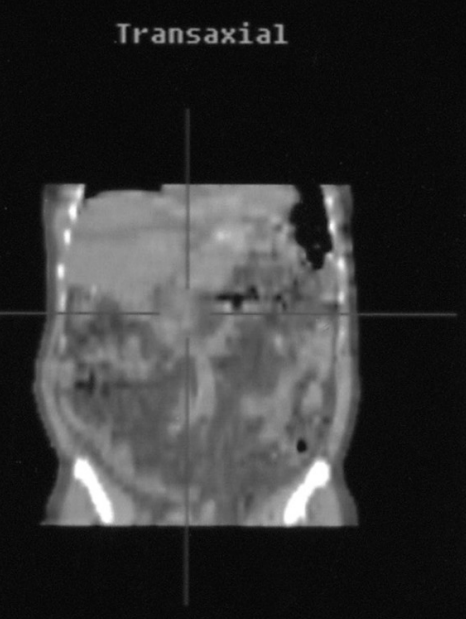

Coronal

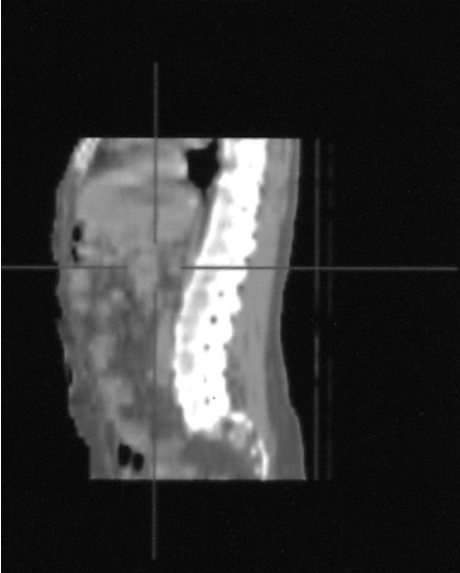

Sagittal
Physiologic

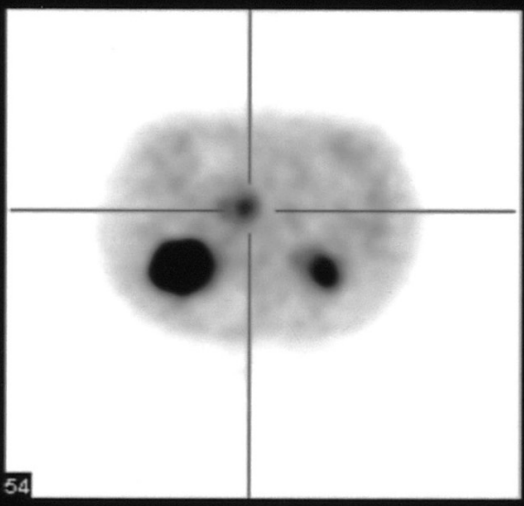

Transaxial

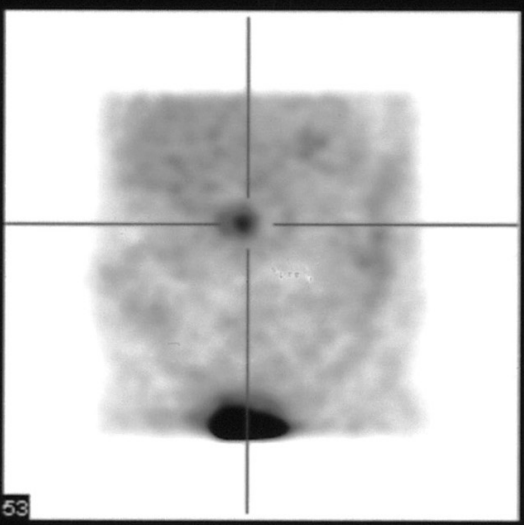

Coronal

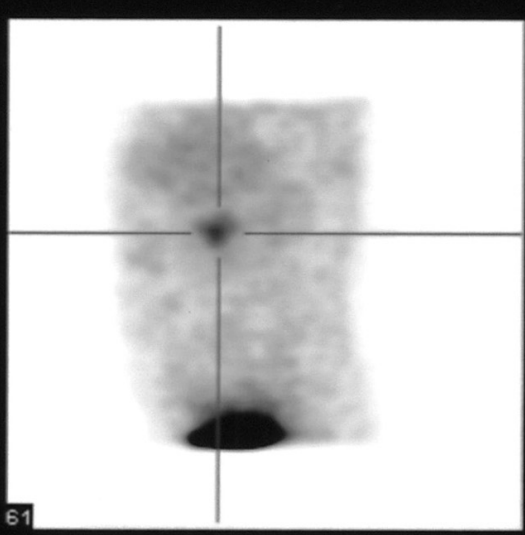

Sagittal
Fusion

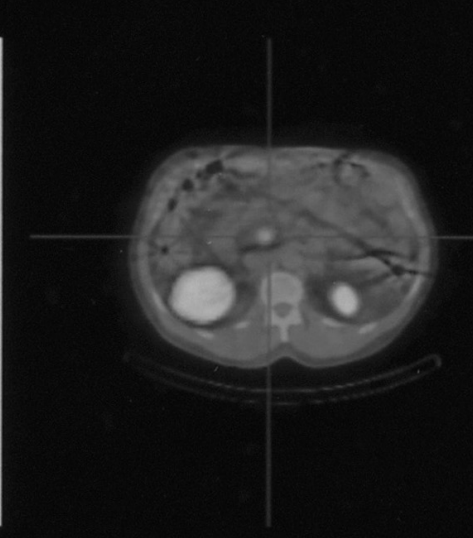

Transaxial

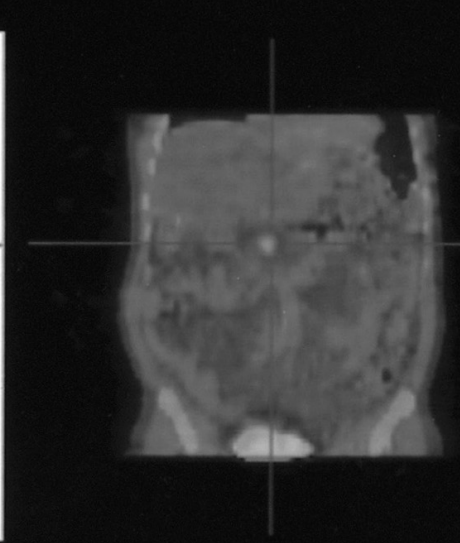

Coronal

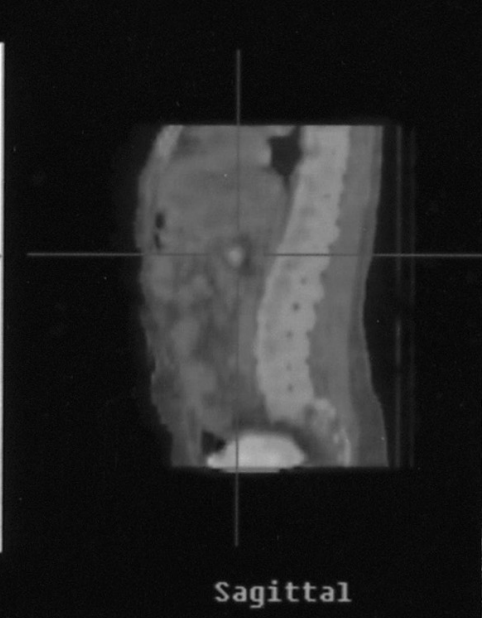

Fig. 1. Selected axial (top), coronal (middle) and sagittal (bottom) CT (left), SPECT (middle) and SPECT/CT (right) images of the abdomen demonstrate focal increased In-111 octreotide receptor uptake (24 hr after injection) in the pancreatic head, with islet cell tumor expressing somatostatin receptor 2 or 5 . 
Kim

and lung tumors can be demonstrated (30) (Fig. 1). Somatostatin receptors such as D2R are members of the Gprotein-coupled receptor family, the largest family of cellsurface receptors involved in signal transduction. Somatostatin and related analogues produce an inhibitory signal which is antiproliferative in cancer cells. The inhibitory effect of SSTR2 on cancer cell proliferation and metastasis is the proposed application as a genetic reporter. Tc-99m P829 (NeoTect) has been used to image lung tumors with SSTR2, 3 or 5, and reporter cassettes inserted in recombinant adenoviral vectors have included SSTR2 alone or in combination with thymidine kinase, cytosine deaminase or GFP. The SSTR2 and TK genes were transferred by mean of the same Ad vector, and both gene

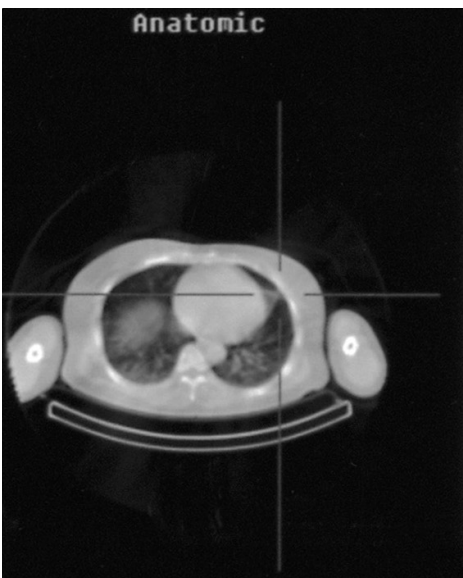

Transaxial

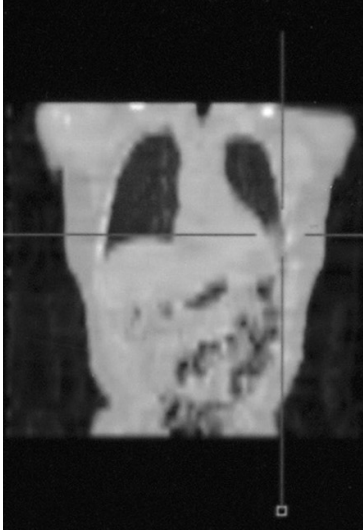

Coronal

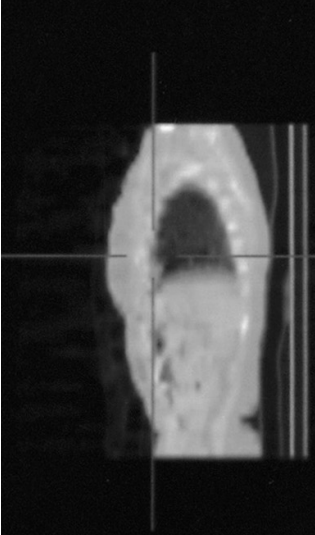

Sagittal

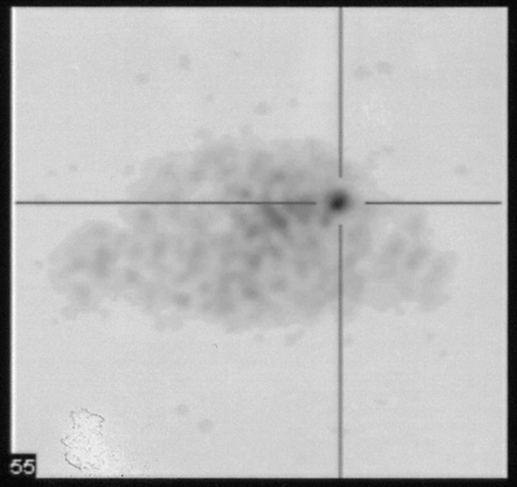

Transaxial

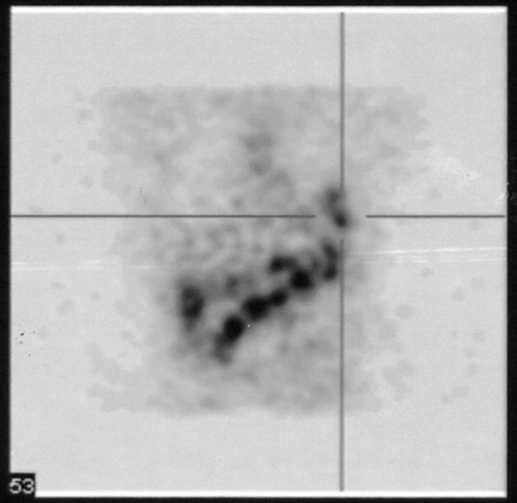

Coronal

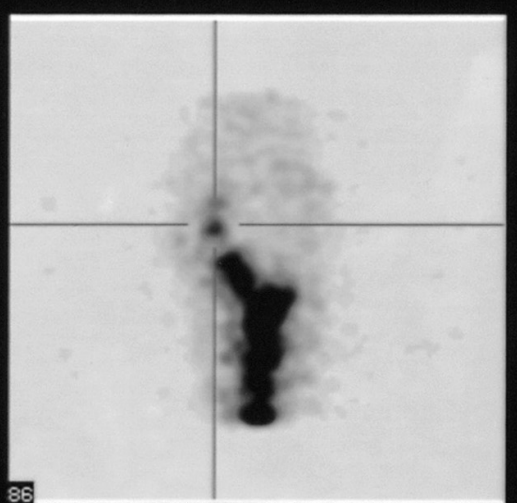

Sagittal
Fusion

Transaxial

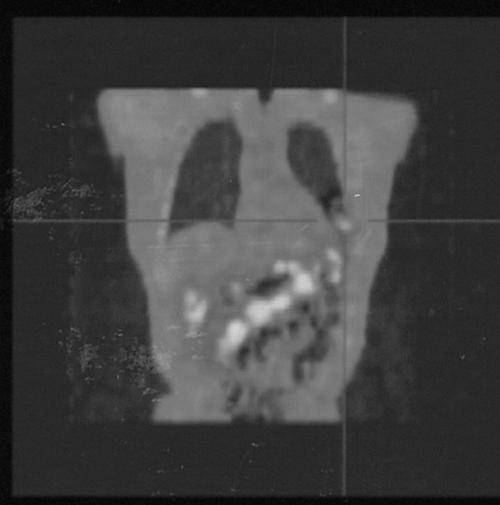

Corona1

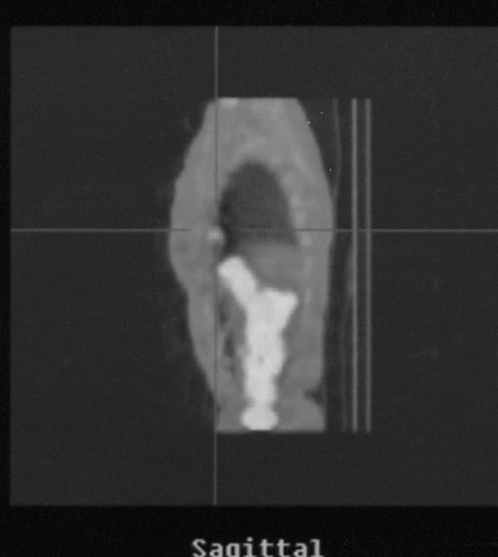

Fig. 2. Selected axial (top), coronal (middle) and sagittal (bottom) CT (left), SPECT (middle) and SPECT/CT (right) images of the chest reveal focal increased uptake of $\mathrm{I}-131$ sodium iodide ( $24 \mathrm{hr}$ after oral ingestion) by the metastatic follicular carcinoma present at the base of the left lung. 
products were simultaneously detected due to internally trapped Tc-99m P2045 and I-131 2'-fluoro-2'-deoxy-1- $\beta$ D-arabinofuranosyl-5-iodo-uracil (FIAU) using a gamma camera which can image multiple radionuclides (31). Octreotide probes conjugated with fluorescent labels can detect somatostatin receptors through light-based imaging (32). An adenoviral vector encoding both SSTR2 and enhanced GFP genes (Ad-SSTR2-GFP) has been developed; the tumor xenograft was visualized after the uptake of Tc99m somatostatin peptide (P2045), using a gamma camera, and also by fluorescent stereomicroscopic imaging (31).

Thyroid iodide uptake is basic to the clinical applications of radioiodine (Fig. 2). Iodine uptake occurs across the membrane of thyroid follicular cells through an active transporter process mediated by N15, a sodium iodide symporter (NIS). The NIS gene is localized on chromosome 9p12-13.2 and encodes a glycoprotein of 643 amino acids. NIS is an integral protein of basolateral membrane of thyroid follicular cells. NIS also transports technetium pertechnetate and rhenium perrhenate in addition to iodide. Thyroid stimulating hormone (TSH) increases iodine uptake, and NIS protein expression is upregulated by TSH. The recent cloning of the NIS gene has improved the better characterization of the molecular mechanisms underlying iodide transport. In papillary and follicular thyroid carcinomas, NIS immunostaining has been positive in only a few tumor cells, and no NIS protein expression has been detected in anaplastic carcinomas. In thyroid carcinomas, decreased NIS levels account for the reduced iodide uptake observed, and by targeting NIS expression in cancer cells which concentrate iodide from plasma, radioiodine therapy can thus be offered. It has been shown that gene transfer of NIS into a variety of cell types confers increased radioiodine uptake by up to several hundredfold that of controls in nonthyroid cancers as well as in thyroid cancer (33). The NIS gene can be transfected to specific cancer cells, and the effect of therapeutic anticancer regimens can be easilly monitored by a gamma camera using radioiodine or technetium pertechnetate. In breast carcinoma, $87 \%$ of 23 invasive carcinomas and $83 \%$ of 6 ductal carcinomas in situ expressed NIS, compared with $23 \%$ of 13 noncancerous tissues adjacent to tumors and none of eight normal breast tissue samples (34). This suggests that during malignant transformation in human breast cancer, NIS is upregulated.

\section{Imaging of Angiogenesis and Apoptosis}

Angiogenesis involves the growth and remodeling of a primitive vascular network into a complex one, and occurs during embryonic development, the female reproductive cycle, wound healing, and hair growth. The vascular network in adults is regulated by angiogenic inducers and inhibitors, the balance of which shifts under pathologic conditions, such as cancer and cardiovascular and immunologic diseases, during which capillary growth is chaotic. Agonist stimulators include vascular endothelial growth factor, basic fibroblast growth factor, angiopoietins, matrix metalloprotease, integrin and cadherin. Endogenous inhibitors include thrombospondin, endostatin, angiostatin, troponin, metallospondin and interleukins. Functional MRI and nu-

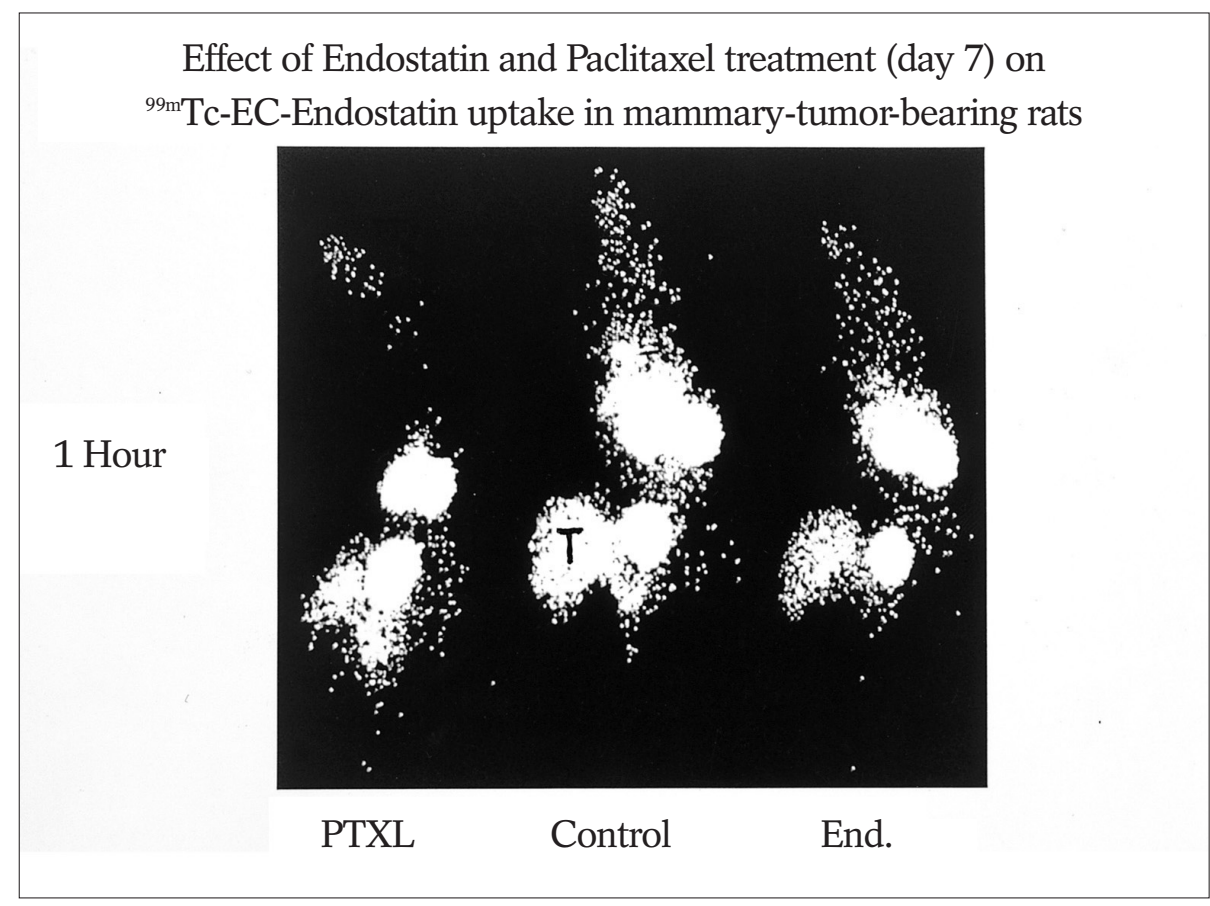

Fig. 3. Planar whole body images of rats bearing breast tumor ( $T$ ) show an uptake of Tc-99m ethylene dicystein (EC)endostatin. Note less uptakes after endostatin (right) and paclitaxel (PTXL) treatments. 
clear techniques generate physiologic parameters such as flow, perfusion, permeability, vascular structure and/or volume fraction based on first-pass or equilibrium contrast enhancement (35). A more specific imaging is to target specific target probes to markers expressed on altered neoendothelial surface of tumor vessels. The phenotypic changes occuring during therapy may not become apparent for weeks or months. Examples include the targeting of endothelial integrin $d \nabla \beta$, using paramagnetic liposomes for MRI, and the targeting of angiogenesis-associated fibronectin using optical probes, radiolabeled peptides adhering to the glycoprotein IIb/IIIa receptor on the surface of activated platelets, and also antibodies against tumor growth factor $\beta$ receptor (1). Tc-99m ethylene dicysteine (EC)-endostatin has been tested in tumor-bearing rats as well as in cancer patients (36) (Fig. 3).

Apoptosis, the process of programmed cell death critical for organ development, tissue homeostasis, and the removal of defective cells without causing concomitant inflammatory response. It depends on the multiple extracellular signals, integration and amplification of signals, and activation of effector proteases called caspases. Apoptosis can be decreased in cancer, autoimmune disease and viral infection, or increased in AIDS, ischemia, stroke and neurodegenerative disorders. There are three pathways of apoptosis (37). The first one involves signaling of a death receptor (eg, Fas or tumor necrosis factor) which then activates caspases. The second one is initiated by the withdrawal of growth factor which leads to the release of cytochrome $\mathrm{C}$ from mitochondria followed by caspase activation, a process controlled by the Bcl-2 protein family. Third one is induced by DNA damage that triggers the p53 pathway of caspase activation. Caspase-mediated proteolysis involving cellular changes such as cell shrinkage, membrane blebbing, chromatin condensation and nuclear fragmentation, is irreversible. Caspase inhibitors may be useful for ischemia, rejection or autoimmune diseases; caspase activator, p53, may help induce efficient killing. During the terminal stages of apoptosis, intracellular phosphatidylserine (PS) is expressed on the cell surface and in annexin contained in tumor cells; annexin $\mathrm{V}$ binds to cells with abnormally expressed PS. Tc-99m HYNIC or EC-annexin V has been used to image the apoptosis occurring in cancer and in transplant rejection (38) (Fig. 4).

\section{Imaging of Drug and Radionuclide Gene Therapy}

The in-vivo testing of drug effectiveness has led to major bottlenecks. Molecular imaging however, can help to determine the pharmacokinetics and pharmacodynamics of

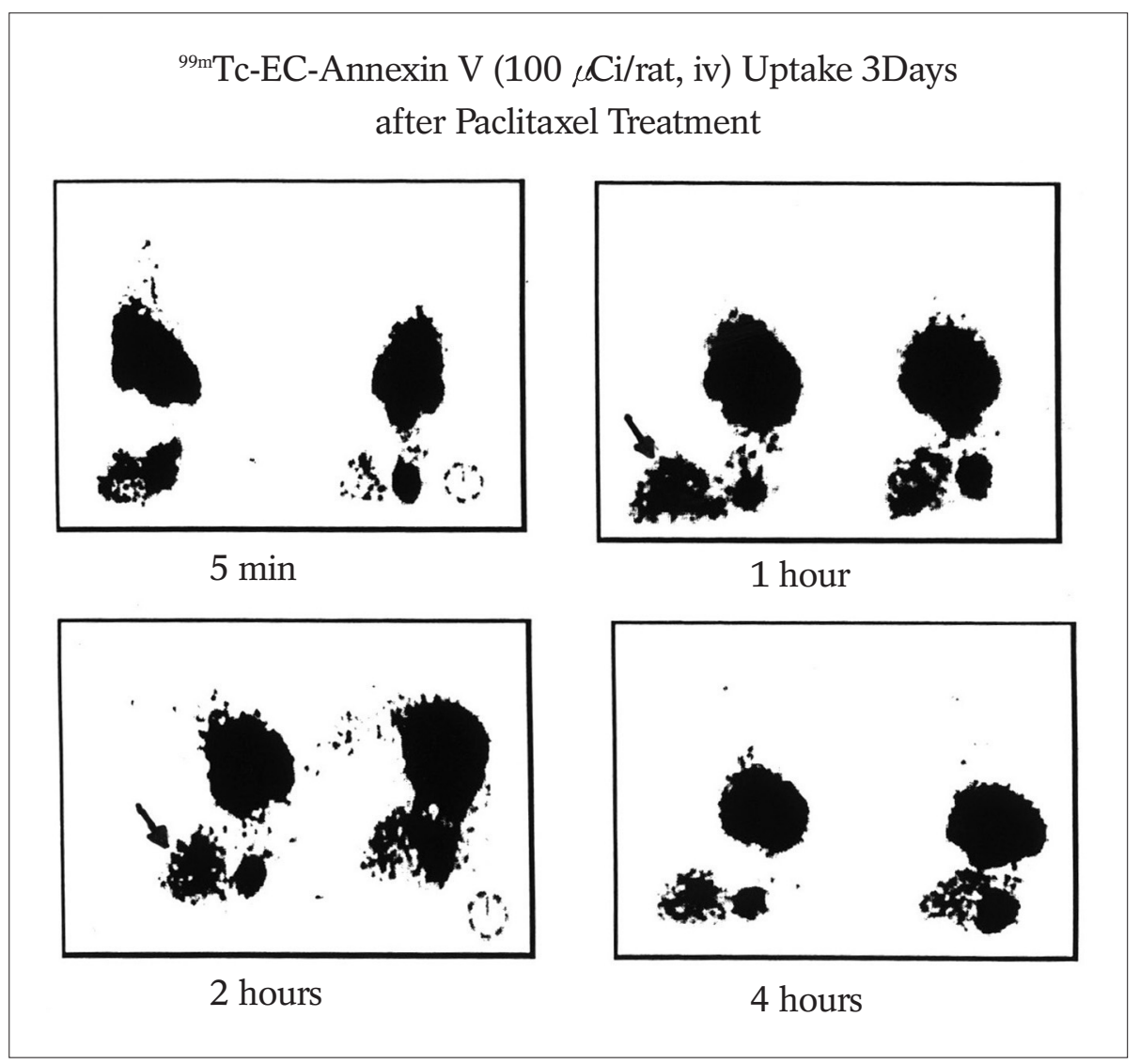

Fig. 4. Planar whole-body images of a breast tumor (arrows) in a rat demonstrate 99mTc-EC-Annexin V uptake after paclitaxel treatment. 
newly developed drugs, and can also facilitate the development of early and objective markers before phenotypic changes become obvious. It is, in addition, useful for probing for a specific molecular target enzyme, receptor or signal transduction pathway against which a therapeutic drug is directed. Imaging is the objective method to assess longterm and often expensive drug testing. It is also helpful to understand delivery barriers for new therapeutic agents.

In addition to the application of somatostatin analogues for imaging, the same and related analogues can be conjugated with therapeutic radionuclides (Y-90, I-131, Sm-153, Lu-177 and Re-188) for therapy. Most efforts are concentrated on therapeutic potential for SSTR-positive neuroendocrine tumors. Y-90 SMT 487 and Re-188 P2045 have been studied to show antitumor effect following Ad-mediated delivery of SSTR2 (39).

Iodide uptake in the NIS-transfected tumors was much higher than that in nontransfected tumors such as anaplastic thyroid cancer, corresponding to NIS mRNA expression in tumors, and can be selectively killed by the I-131 sodium iodide. Combining the targeting and expression of NIS gene together with radioiodine treatment can be also used to treat non-thyroid cancers such as hepatoma, melanoma, colon and ovarian adenocarcinomas (33). NIS gene transfer using tissue-specific promoters provides a way of selectively targeting NIS gene to malignant cells, thereby maximizing tissue-specific cytotoxicity and minimizing toxic side effects on nonmalignant cells.

\section{Conclusion}

Molecular imaging helps visualize the cellular and/or molecular process in living tissues. Recent advances in imaging technologies and gene transfer strategies optimize gene therapy trials. The development of molecular imaging probes is most important, and reporter genes are very useful to monitor the delivery, magnitude and time variation of therapeutic gene transfer. One of the goals in molecular imaging research is the development of universal imaging genes. Such generic reporters could then be used to facilitate direct imaging of promoter activity over time. Molecular imaging technologies must have the capability of non-invasively detecting, measuring, and reporting the expression of multiple meaningful genes simultaneously. We are just scratching the surface of complex biochemical pathways ongoing in biological systems. Micro-PET, SPECT, MRI and CT, as well as optical and bioluminescence cameras are helpful instruments to generate the results of successful innovation and feasibility studies in animals.

\section{References}

1. Weissleder R, Mahmood U. Molecular imaging. Radiology 2001;219:316-333

2. Pennisi E. Human genome: finally, the book of life and instructions for navigating it. Science 2000;288:2304-2307

3. Marshall E. Gene therapy on trial. Science 2000;288:951-957

4. O'Malley BW Jr, Couch ME. Gene therapy principles and strategies for head and neck cancer. Adv Otorhinolaryngol 2000;56:279-288

5. Wilson JM. Adenoviruses as gene-delivery vehicles. $N$ Engl J Med 1996;334:1185-1187

6. Robbins PD, Ghivizzani SC. Viral vectors for gene therapy. Pharmacol Ther 1998;80:35-47

7. Chmura SJ, Advani SJ, Kufe DW, Weichselbaum RR. Strategies for enhancing viral-based gene therapy using ionized radiation. Radiat Oncol Investig 1999;7:261-269

8. Chen EA. A comtemporary review of gene therapy and its applications. Applied Radiology 2001;30:36-48

9. Boerman OC, Oyen WJ, Corstens FH. Progress in gene therapy: seeing is believing. J Nucl Med 2001;42:1235-1237

10. Tuting T, Storkius WJ, Lotze MT. Gene-based strategies for the immunotherapy of cancer. J Mol Med 1997;75:478-491

11. Arai H, Gordon D, Nabel EG, Nabel GJ. Gene transfer of Fas ligand induces tumor regression in vivo. Proc Natl Acad Sci U.S.A. 1997;94:13862-13867

12. Roth JA, Nguyen D, Lawrence DD, et al. Retrovirus-mediated wild-type p53 gene transfer to tumors of patients with lung cancer. Nature Med 1996;2:985-991

13. Ogawa N, Fujiwara T, Kagawa S, et al. Novel combination therapy for human colon cancer with adenovirus-mediated wildtype p53 gene transfer and DNA-damaging chemotherapeutic agent. Int J Cancer 1997;73:367-370

14. Barnes MN, Deshane JS, Rosenfeld M, Siegal GP, Curiel DT, Alvarez RD. Gene therapy and ovarian cancer: a review. Obstet Gynecol 1997;89:145-155

15. Feldman AL, Libutti SK. Progress in antiangiogenic gene therapy of cancer. Cancer 2000;89:1181-1194

16. Ramesh R, Marrogi AJ, Munshi A, Abboud CN, Freeman SM. In vivo analysis of the bystander effect: a cytokine cascade. Exp Hematol 1996;24:829-838

17. Rosenberg RA. Cancer vaccines based on the identification of genes encoding cancer regression antigens. Immunol Today 1997;18:175-182

18. Guinan EC, Gribben JG, Boussiotis VA, Freeman GJ, Nadler LM. Pivotal role of the B7/CD28 pathway in transplantation tolerance and tumor immunity. Blood 1994;84:3261-3282

19. Corr M, Tighe H, Lee D, et al. Costimulation provided by DNA immunization enhances antitumor immunity. J Immunol 1997; 159:4999-5004

20. Freeman SM, Whartenby KA, Freeman JL, Abboud CN, Marrogi A. In situ use of suicide genes for cancer therapy. Semin Oncol 1996;23:31-45

21. Haberkorn U, Oberdorfer F, Gebert J, et al. Monitoring gene therapy with cytosine deaminase: in vitro studies using 3H-5fluorocytosine. J Nucl Med 1996;37:87-94

22. Thomas JW, Kuo MD, Chawla M, et al. Vascular gene therapy. RadioGraphics 1998;18:1373-1394

23. Hogemann D, Basilion JP. Seeing inside the body: MR imaging of gene expression. Eur J Nucl Med 2002;29:400-408

24. Misslitz A, Mottram JC, Overath P, Aebischer T. Targeted integration into a rRNA locus results in uniform and high level ex- 
pression of transgenes in Leishmania amastigotes. Mol Biochem Parasitol 2000;107:251-261

25. Seul KH, Beyer EC. Mouse connexin 37: gene structure and promoter analysis. Biochim Biophys Acta 2000;1492:499-504

26. Gambhir SS, Bauer E, Black ME, et al. A mutant herpes simplex virus type 1 thymidine kinase reporter gene shows improved sensitivity for imaging reporter gene expression with positron emission tomography. Proc Natl Acad Sci U.S.A. 2000;97:27852790

27. Haberkorn U, Oberdorfer F, Gebert J, et al. Monitoring gene therapy with cytosine deaminase: in vitro studies using 3H-5fluorocytosine. J Nucl Med 1996;37:87-94

28. MacLaren DC, Gambhir SS, Satyamurthy N, et al. Repetitive, non-invasive imaging of the dopamine D2 receptor as a reporter gene in living animals. Gene Ther 1999;6:785-791

29. Iyer M, Barrio JR, Namavari M, et al. 8-[18F] fluoropenciclovir: an improved reporter probe for imaging HSV1-tk reporter gene expression in vivo using PET. J Nucl Med 2001;42:96-105

30. Zinn KR, Chaudhuri TR. The type 2 human somatostatin receptor as a platform for reporter gene imaging. Eur J Nucl Med 2002;29:388-399

31. Zinn KR, Chaundhuri TR, Krasnykh VN, et al. Gamma camera dual imaging with a somatostatin receptor and thymidine kinase after gene transfer with a bicistronic adenovirus in mice. Radiology 2002;223:417-425
32. Becker A, Hessenius C, Licha K, et al. Receptor-targeted optical imaging of tumors with near-infrared fluorescent ligands. Nat Biotechnol 2001;19:327-331

33. Chung J-K. Sodium iodide symporter: its role in nuclear medicine. J Nucl Med 2002;43:1188-1200

34. Tazebay UH, Wapnir IL, Levy O, et al. The mammary gland iodide transporter is expressed during lactation and in breast cancer. Nat Med 2000;6:871-878

35. Turetschek K, Preda A, Floyd E, et al. MRI monitoring of tumor response following angiogenesis inhibition in an experimental human breast cancer model. Eur J Nucl Med 2003;30:448-455

36. Yang DJ, Kim K-D, Schechter NR, et al. Assessment of antiangiogenic effect using Tc-99m EC-endostatin. Cancer Biother Radiopharm 2002;17:233-245

37. Schmitt CA, Lowe SW. Apoptosis and therapy. J Pathol 1999;187:127-137

38. Ogura Y, Krams SM, Martinez OM, et al. Radiolabeled annexin $\mathrm{V}$ imaging: diagnosis of allograft rejection in an experimental rodent model of liver transplantation. Radiology 2000;214:795800

39. Rogers BE, Zinn KR, Lin CY, Chaudhuri TR, Buchsbaum DJ. Targeted radiotherapy with Y-90 SMT 487 in mice bearing human non-small cell lung tumor xenografts induced to express human somatostatin receptor type 2 with an adenoviral vector. Cancer 2002;94:1298-1305 